\title{
CELULITIS FACIAL ODONTOGÉNICA SEVERA INFRECUENTE CAUSADA POR CITROBACTER FREUNDII PRODUCTORA DE AMPC EN UN PACIENTE CON DIABETES MELLITUS 2. REPORTE DE CASO
}

\author{
Camilo Eslava ${ }^{1}$, Sergio Castellanos ${ }^{1}$, Elías Franco Pretto ${ }^{2}$, Valentina Sánchez ${ }^{2}$, Iván MÉndez
}

${ }^{1}$ Especialista en Cirugía Oral y Maxilofacial del Hospital Militar Central - Universidad Militar Nueva Granada

${ }^{2}$ Residente en Cirugía Oral y Maxilofacial del Hospital Militar Central - Universidad Militar Nueva Granada

${ }^{3}$ Docente Enfermedades Infecciosas. Grupo Patogenicidad Microbiana. Facultad de Medicina - UMNG

\begin{abstract}
Resumen
El manejo de la celulitis facial odontogénica no deja de ser un tema controversial en el campo de la cirugía oral y maxilofacial; los principios quirúrgicos y terapéuticos han sido sometidos a modificaciones basadas en los hallazgos clínicos, imagenológicos y microbiológicos a través del tiempo. En pacientes con diabetes mellitus 2 se incrementa el riesgo a sufrir infecciones bacterianas oportunistas con tiempos de hospitalización más prolongados que la población no diabética. La literatura es clara estableciendo las diferencias clínicas y microbiológicas de la celulitis facial odontogénica en este grupo de pacientes, sin embargo, no existe un protocolo médico quirúrgico destinado a ellos. El microorganismo comúnmente aislado es Klebsiella pneumoniae, mientras Citrobacter freundii es inusual en las infecciones odontogénicas, su capacidad para producir betalactamasas de amplio espectro $(\mathrm{AmpC})$ le permite bloquear la acción de los antibióticos de uso empírico en Cirugía Oral y Maxilofacial. A continuación, presentamos el caso de una paciente de 61 años con diabetes Mellitus tipo 2 y celulitis facial odontogénica por Citrobacter freundii productora de AmpC.
\end{abstract}

Palabras clave: Diabetes mellitus, celulitis, Citrobacter freundii, betalactamasas AmpC.

\section{UNCOMMON SEVERE ODONTOGENIC FACIAL CELLULITIS CAUSED BY AMPC-PRODUCING CITROBACTER FREUNDII IN A PATIENT WITH TYPE 2 DIABETES MELLITUS. CASE REPORT}

\begin{abstract}
The management of odontogenic facial cellulitis is still a controversial issue in the field of Oral and Maxillofacial Surgery. Surgical and therapeutic principles have undergone modifications based on clinical findings, imaging and microbiological over time. In patients with type 2 Diabetes Mellitus the risk of opportunistic bacterial infections is increased thus suffering longer hospitalization periods than the nondiabetic population. The literature is clear by setting the clinical and microbiological differences of odontogenic facial cellulitis in this group of patients, but there is no surgical medical protocol for them. Klebsiella pneumoniae is the most common microorganism isolated while Citrobacter freundii is unusual in relation to oral infections; their ability to produce ESBLs $(\mathrm{AmpC})$ allows them to block the action of empirical antibiotics used in Maxillofacial Surgery. We present the case of a 61 year old patient with type 2 Diabetes Mellitus and odontogenic facial cellulitis caused by AmpCproducing Citrobacter freundii.
\end{abstract}

Key words: Diabetes Mellitus, Cellulitis, Citrobacter freundii, AmpC beta-lactamases.

* Correspondencia: Elías Franco Pretto. Correo electrónico: elias.pretto@gmail.com. Dirección Correspondencia: Tr. 3 No 49-00. Servicio de Cirugía Oral y Maxilofacial. Hospital Militar Central, Bogotá, Colombia. 


\title{
CELULITE FACIAL ODONTOGÊNICA SEVERA INFREQUENTE CAUSADA POR CITROBACTER FREUNDII PRODUTORA DE AMPC EM UM PACIENTE COM DIABETES MELLITUS 2. RELATÓRIO DE CASO
}

\begin{abstract}
Resumo
O tratamento da celulite facial odontogênica não deixa de ser um tema controverso no campo da Cirurgia Oral e Maxilo-facial; os princípios cirúrgicos e terapêuticos foram submetidos a modificações baseadas nos descobrimentos clínicos, imagenológicos e microbiológicos através do tempo. Em pacientes com Diabetes Mellitus 2 aumenta o risco de sofrer infecções bacterianas oportunistas com tempos de hospitalização mais prolongados que na população não diabética. A literatura é clara estabelecendo as diferenças clínicas e microbiológicas da Celulite Facial Odontogênica neste grupo de pacientes; porém, não existe um protocolo médico cirúrgico destinado a eles. $\mathrm{O}$ microrganismo comunmente isolado é o Klebsiella pneumoniae, enquanto que o Citrobacter freundii é inusual nas infecções odontogênicas, sua capacidade para produzir beta-lactamases de amplo espectro (AmpC) lhe permite bloquear a ação dos antibióticos de uso empírico em Cirurgia Oral e Maxilo-facial. A seguir apresentamos o caso de uma paciente de 61 anos com Diabetes Mellitus tipo 2 e celulite facial odontogênica por Citrobacter freundii produtora de AmpC.
\end{abstract}

Palavras chave: Diabetes Mellitus, Celulitis, Citrobacter freundii, beta-lactamases AmpC.

\section{Introducción}

La celulitis facial odontogénica es un problema común en el campo de la Cirugía Maxilofacial. A pesar del conocimiento de los principios quirúrgicos de manejo; la aparición de cepas resistentes a los antimicrobianos puede prolongar el tiempo de resolución del cuadro y modificar el manejo hospitalario (1).

La diabetes mellitus forma parte de un síndrome metabólico caracterizado por elevación de niveles plasmáticos de glucosa (>126mg/dl) causado por ausencia parcial o completa de insulina. Esta enfermedad incrementa el riesgo del paciente a sufrir infecciones bacterianas a expensas de la disfunción en la capacidad bactericida de los polimorfonucleares, de la inmunidad celular y de la activación del sistema de complemento $(1,2)$.

Existen estudios que comparan las características clínicas de las infecciones maxilofaciales entre pacientes diabéticos y no diabéticos en términos de severidad, tasa de complicaciones y biota. Los resultados demuestran que los pacientes diabéticos presentan cuadros infecciosos más severos, con una microbiota distinta de los pacientes no diabéticos (3-7).
Klebsiella pneumoniae ha sido reportado como el microorganismo más común en los casos de celulitis facial odontogénica en pacientes diabéticos, mientras Citrobacter freundii es inusual en las infecciones odontogénicas. Ambos son bacilos gram negativos entéricos oportunistas capaces de producir betalactamasas de distintas clases siendo las del tipo AmpC frecuentes en cepas de Citrobacter freundii, Enterobacter spp, Morganella morganii y Serratia marcescens complicando el manejo farmacológico de las infecciones asociadas a estos microorganismos $(5,6)$.

El mecanismo predominante de resistencia a los betalactámicos en las bacterias gram-negativas es la síntesis de betalactamasas. La AmpC es una $\beta$ lactamasa de tipo I codificada por plásmido o en cromosoma. El gen $A m p C$ otorga resistencia a aminopenicilinas, cefalosporinas de primera, segunda y tercera generación, cefamicinas y betalactámicos más inhibidores de betalactamasas $(8,9)$.

A continuación se realiza reporte de un caso clínico de celulitis facial odontogénica severa con aislamiento de Citrobacter freundii productora de AmpC y Klebsiella pneumoniae sensible a la mayoría de los betalactámicos. 


\section{Caso clínico}

Paciente femenina de 61 años de edad quien acude al servicio de urgencias del Hospital Militar Central el día 10 de noviembre de 2011, tras presentar un cuadro clínico de 10 días de evolución consistente en dolor orofacial y edema persistente en hemicara izquierda posterior a exodoncia extrainstitucional de diente 38 en inclusión submucosa horizontal el día 1 de noviembre de 2011, acompañado de malestar general, disfagia y fiebre subjetiva no cuantificada. Dentro de los antecedentes médicos de relevancia, la paciente reporta múltiples comorbilidades: diabetes mellitus tipo 2, hipertensión arterial esencial, hipotiroidismo, neuropatía diabética; farmacológicos: levotiroxina 75 $\mu \mathrm{g}$, enalapril $20 \mathrm{mg}$, nifedipino $30 \mathrm{mg}$, omeprazol 20 $\mathrm{mg}$, atorvastatina $10 \mathrm{mg}$, todos éstos a dosis diarias, adicionalmente insulina glargina 24 UI subcutáneas cada mañana.

El manejo farmacológico que siguió la paciente de manera extra-institucional incluyó amoxicilina 500 mg vía oral cada 8 horas por 7 días. Sin embargo, consultó nuevamente a su dispensario el día octavo de su posoperatorio por incremento del edema, rubefacción y disfagia. Se inició esquema antibiótico con clindamicina $300 \mathrm{mg}$ vía oral cada 6 horas; la paciente reporta haber cumplido con una sola dosis de dicho antibiótico.

Al décimo día tras no haber mejoría del cuadro clínico, es remitida del dispensario al servicio de urgencias del Hospital Militar Central, donde es valorada por el servicio de Cirugía Oral y Maxilofacial. Al examen físico se encontraba en buen estado general, álgida y somnolienta. Al examen facial se evidenciaba edema en el tercio inferior de hemicara izquierda, indurado, doloroso a la palpación circunscrito a la región submandibular y retroauricular izquierda. Al examen intraoral se palpaba induración de piso de boca de ese mismo lado, sin elevación el mismo, con evidencia de secreción purulenta de color amarillo sin fetidez. Se procedió a tomar muestra y se envió a cultivo y antibiograma, se ordenó tomografía computarizada simple de cara y cuello que muestra imágenes circunscritas hipodensas irregulares compatibles con colección en región de espacio pterigomandibular izquierdo, Figura 1.

Los signos vitales de ingreso al Hospital Militar Central y resultados de los exámenes paraclínicos se detallan en la tabla 1. Se inició manejo con líquidos endovenosos, analgesia y tratamiento antimicrobiano con clindamicina $600 \mathrm{mg}$ cada 6 horas, ciprofloxacina 400 mg cada 12 horas de acuerdo con los lineamientos del servicio de Medicina Interna.

A las 48 horas posteriores a la toma de la muestra, el reporte preliminar del cultivo muestra crecimiento de cocobacilos gramnegativos; se modifica el manejo a ampicilina sulbactam $3 \mathrm{~g}$ endovenosos cada 6 horas conjugado con clindamicina $600 \mathrm{mg}$ endovenosos cada 6 horas a su tercer día de hospitalización. Al día octavo se obtienen los resultados definitivos del cultivo y antibiograma (tabla 2); arrojando crecimiento escaso de cocobacilos gramnegativos, crecimiento moderado de Klebsiella pneumoniae, Aggregatibacter (Haemophilus) segni y crecimiento escaso de Citrobacter freundii productora de AmpC. Con base en el hallazgo, se interconsulta al servicio de Infectología para manejo integral quienes sugirieron suspender clindamicina e iniciar meropenem $1 \mathrm{~g}$ endovenoso cada 8 horas por 14 días, continuando manejo conjugado con ampicilina sulbactam por 10 días.

Se llevó estricto control de la glicemia cada 6 horas, dieta hipoglúcida e hiposódica, líquidos endovenos (dextrosa en agua destilada al 10\% $20 \mathrm{cc}$ en caso de no tolerar la vía oral) y los fármacos para control de las comorbilidades.

La paciente fue llevada a cirugía en su cuarto día de manejo antibiótico definitivo; se realiza incisión de $3 \mathrm{~cm}$ de longitud, a $3 \mathrm{~cm}$ desde el borde inferior mandibular, mediante disección roma subplastimal se procede a realizar drenaje, obteniendo aproximadamente $10 \mathrm{cc}$ de contenido purulento de color amarillo sin fetidez.

El cuadro clínico presenta resolución completa al día 14 de haber iniciado el manejo con meropenem y ampicilina sulbactam, por lo que se da salida a la paciente, con controles por el servicio de cirugía oral y maxilofacial.

\section{Discusión}

Existen muchos reportes que establecen las diferencias entre pacientes diabéticos y no diabéticos en relación con la presentación clínica y evolución de la celulitis facial odontogénica. El control estricto de los niveles séricos de glucosa es de suma importancia para el 


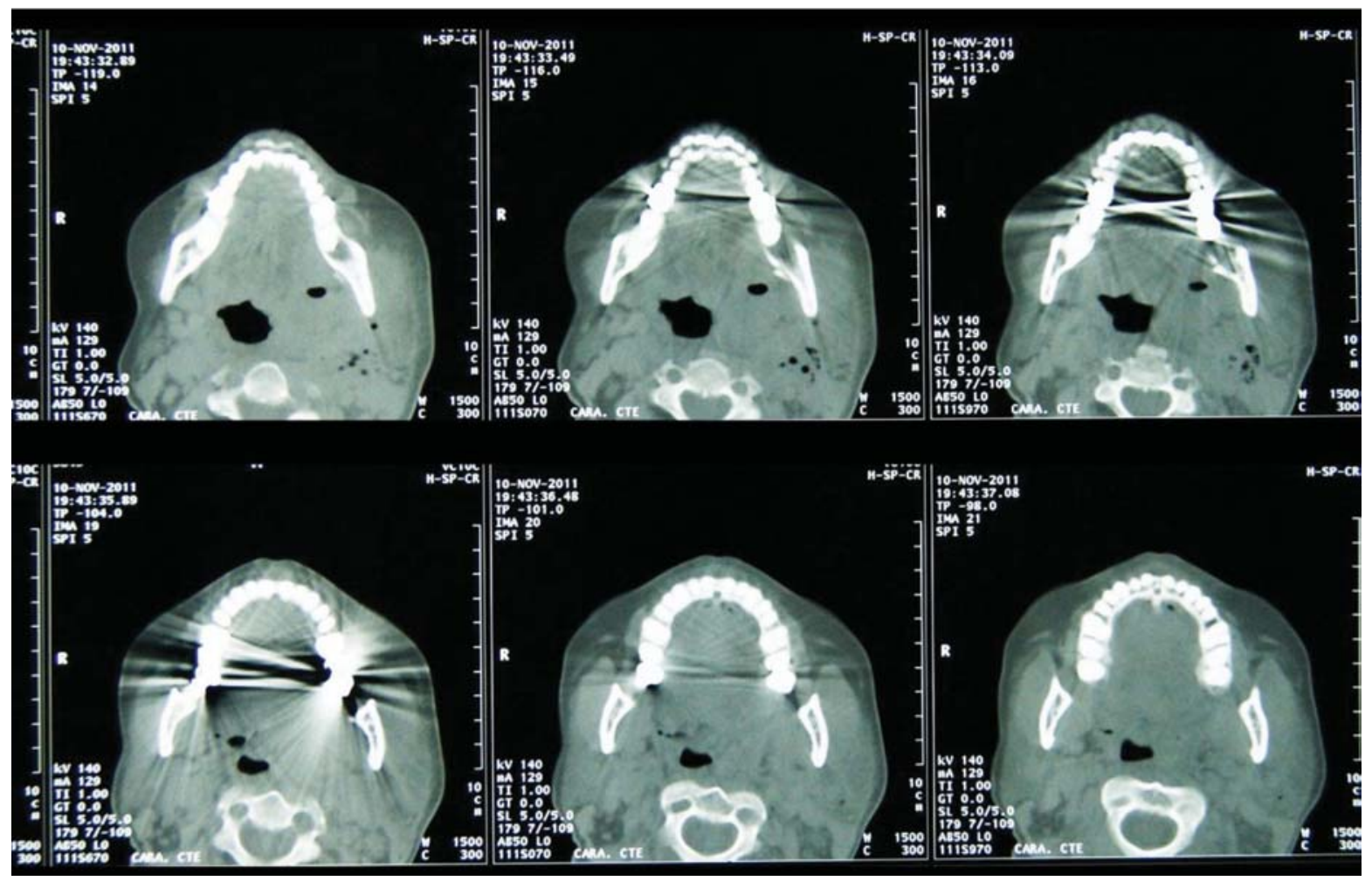

Figura 1. Cortes axiales de tomografía computarizada simple de cara y cuello. Se observan imágenes hipodensas compatibles con colección gaseosa en espacio pterigomandibular izquierdo y aumento de volumen de tejidos blandos concomitante con desviación de vía aérea hacia la derecha.

Tabla 1. Resultados de los exámenes paraclínicos y signos vitales al ingreso el día 10 de noviembre de 2011.

\begin{tabular}{|l|c|c|c|}
\hline \multicolumn{1}{|c|}{ Examen } & Resultado & Unidades & Valores de referencia \\
\hline Signos Vitales & & & \\
\hline Tensión Arterial & $150 / 70$ & $\mathrm{mmHg}$ & \\
\hline Frecuencia Cardíaca & 80 & $\neq \mathrm{pm}$ & \\
\hline Frecuencia Respiratoria & 16 & $\neq \mathrm{rpm}$ & \\
\hline Gases arteriales & & & $7.35-7.45$ \\
\hline $\mathrm{pH}$ & 7.47 & & $70-90$ \\
\hline $\mathrm{pCO}^{2}$ & 61.3 & $\mathrm{mmHg}$ & $20-30$ \\
\hline $\mathrm{HCO}_{3}$ & 22.7 & $\mathrm{mmHg}$ & \\
\hline
\end{tabular}

†Ipm latidos por minuto, ¥rpm respiraciones por minuto

adecuado control de las infecciones profundas del cuello y la reducción del período de hospitalización, así mismo, permite proteger la vía aérea reduciendo la necesidad de realizar traqueostomía. La paciente de este reporte cursó con niveles séricos de glucosa normales durante su período de hospitalización. Sin embargo, existen reportes que afirman que el valor elevado de hemoglobina glicosilada es un predictor importante de infección posoperatoria en cirugía cardiotorácica (4).

Los cultivos y prueba de susceptibilidad fueron fundamentales para la detección de un microorganismo inusual como lo es Citrobacter freundii productor 
Tabla 2. Resultados de la prueba de susceptibilidad.

\begin{tabular}{|c|c|c|}
\hline Microorganismo & Antibiótico & Sensibilidad \\
\hline \multirow[t]{10}{*}{ Citrobacter freundii* } & Amikacina & Sensible $<=2^{\neq}$ \\
\hline & Ampicilina & Resistente 4 \\
\hline & Ampicilina Sulbactam & Resistente $<=2$ \\
\hline & Cefalotina & Resistente 32 \\
\hline & Cefepime & Sensible $<=1$ \\
\hline & Cefoxitina & Resistente $>=64$ \\
\hline & Ciprofloxacina & Sensible $<=0.25$ \\
\hline & Gentamicina & Sensible $<=1$ \\
\hline & Imipenem & Sensible $<=1$ \\
\hline & Meropenem & Sensible $<=0,25$ \\
\hline \multirow[t]{10}{*}{ Klebsiella pneumoniae } & Amikacina & Sensible $<=2$ \\
\hline & Ampicilina & Resistente 16 \\
\hline & Ampicilina Sulbactam & Sensible 4 \\
\hline & Cefalotina & Sensible $<=2$ \\
\hline & Cefepime & Sensible $<=1$ \\
\hline & Cefotaxime & Sensible $<=1$ \\
\hline & Ciprofloxacina & Sensible $<=0.25$ \\
\hline & Gentamicina & Sensible $<=1$ \\
\hline & Imipenem & Sensible $<=1$ \\
\hline & Meropenem & Sensible $<=0.25$ \\
\hline
\end{tabular}

*Productora de AmpC,$\neq \mathrm{CIM}(\mu \mathrm{g} / \mathrm{mL}) ., \neq \mathrm{CIM}(\mu \mathrm{g} / \mathrm{mL})$.

de AmpC y de Klebsiella pneumoniae que ha sido reportado como el microorganismo más frecuente en infecciones odontogénicas y no odontogénicas de espacios profundos del cuello (6).

Citrobacter freundii es un bacilo gram negativo motil, entérico, anaerobio facultativo, cuyo hábitat incluye el medio ambiente (tierra, agua, unidad de tratamiento de aguas servidas), alimentos y el tracto gastrointestinal de animales y humanos (10).

Por ser un patógeno oportunista, C. freundii es considerado responsable de infecciones oportunistas de importancia en pacientes con un sistema inmune debilitado. Este microorganismo es conocido por causar una variedad de infecciones nosocomiales de tracto respiratorio y urinario, sistema circulatorio y otros sitios normalmente estériles (11).
Las especies de Citrobacter han sido asociadas de manera frecuente a pacientes con infecciones nosocomiales con estadía hospitalaria prolongada. Nuevas cepas de Citrobacter freundii resistentes a la ceftriaxona (CRCF) han sido aisladas de pacientes con comorbilidades como el virus de inmunodeficiencia humana - síndrome de inmunodeficiencia adquirida (VIH-SIDA), enfermedad periférica vascular y enfermedad cerebrovascular. Hay reportes que afirman que las fluoroquinolonas no son efectivas contra los CRCF (12).

Cuando estas cepas portadoras del plásmido que codifica la beta lactamasa AmpC se exponen a los carbapenémicos y cefamicinas, muestran sensibilidad a éstos (13).

La AmpC es una betalactamasa del grupo I que se encuentran de manera natural en microorganismos como Enterobacters pp., Providencia spp., 
Morganella morganii, Serratia marcenscens, Citrobacter freundii, Hafnia alvei. AmpC es de naturaleza plasmídica otorgándoles resistencia a las aminopenicilinas, cefalosporinas de primera generación, y aminopenicilinas combinadas con inhibidores de betalactamasas. Una característica de las cepas productoras de AmpC es su sensibilidad a las cefalosporinas de cuarta generación y los carbapenémicos como el meropenem, siendo éstos últimos de elección contra cepas productoras de AmpC (14).

Por su parte K. pneumoniaees una bacteria gramnegativa en forma de bastón, productora de ureasa, localizada comúnmente en el tracto gastrointestinal y aislada en manos del personal hospitalario. La razón de su patogenicidad es la gruesa cápsula que rodea a esta bacteria, al igual que $C$. freundii, su hábitat incluye también el agua, unidades de tratamiento de aguas servidas y suelo. Las infecciones ocasionadas por K. pneumoniae incluyen la neumonía, septicemia e infección en tejido blando (15-17).

Klebsiellap neumoniae además de ser detectado como el causal de infecciones severas del tracto urinario, neumonía nosocomial e infecciones intraabdominales es causante de neumonía adquirida en la comunidad (18). Un hecho importante, es la capacidad de K. pneumoniae de producir enzimas KPC (del inglés Klebsiella pneumonia ecarbapenemases), codificadas en plásmidos; el espectro de hidrólisis de estas enzimas incluyen a cefotaxime y los carbapenémicos. Aunque las betalactamasas del tipo KPC son encontradas de manera predominante en $K$. pneumoniae, existen reportes de estas enzimas en Enterobacter spp y Salmonella spp. La cepa aislada en la paciente reveló sensibilidad a cefotaxime, meropenem e imipenem (19).

\section{Conclusiones}

La celulitis facial odontogénica es una condición que suele responder de manera satisfactoria al manejo antibiótico empírico con antimicrobianos betalactámicos como la penicilina y lincosamidas como la clindamicina, sin embargo, la diabetes mellitus tipo 2 predispone al paciente a enfrentarse a infecciones oportunistas de mayor severidad y progresión acelerada.

Este reporte de caso enfatiza la importancia de interpretar de manera acertada la prueba de susceptibilidad a los antibióticos. En pacientes con diabetes mellitus tipo 2 constituye una herramienta eficaz y oportuna en el establecimiento de la terapia antibiótica definitiva. La microbiota en la celulitis facial odontogénica ha sido estudiada y los microorganismos mas frecuentes han sido identificados, sin embargo, cuando se aíslan agentes infecciosos causales poco comunes en estas infecciones como Citrobacter freundii, la estadía hospitalaria se prolonga debido a la falla en la terapéutica inicial y el tiempo necesario para su identificación. Adicionalmente, el riesgo de adquirir una infección nosocomial por el período prolongado de hospitalización que podría comprometer la adecuada evolución del paciente.

En vista de los hallazgos microbiológicos y clínicos, el manejo empírico hospitalario sugerido para la celulitis facial odontogénica en la literatura actual podría no ser el adecuado en pacientes con diabetes mellitus. Actualmente no existe un protocolo de manejo antibiótico para este grupo de pacientes. Éste es el primer caso de celulitis facial odontogénica con aislamiento de Citrobacter freundii reportado en la literatura.

Corresponde al cirujano oral y maxilofacial, reconocer la importancia de la anamnesis que junto al examen clínico e interpretación de las ayudas diagnósticas lleven al manejo adecuado de las infecciones odontogénicas en pacientes que padecen diabetes mellitus tipo 2 .

\section{Agradecimientos}

Al laboratorio de Microbiología del Hospital Militar Central.

\section{Referencias bibliográficas}

1. Flynn T. What are the Antibiotics of Choice for Odontogenic Infections, and How Long Should the Treatment Course Last? Oral MaxillofacSurgClin N Am. 2011 November; 23(4): 519-536.

2. Rao DD, Desai A, Kulkarni RD, Gopalkrishnan K, Rao B. Comparison of maxillofacial space infection in diabetic and non diabetic patients. Oral Surg Oral Med Oral Pathol Oral RadiolEndod. 2010 April; 110: e7-e12.

3. Muller LMA, Gorter KJ, Hak E, Goudzwaard WL, Schellevis FG, Hoepelman A et al. Increased risk of common infections in patients with type 1 and type 2 diabetes mellitus. Clin Infect Dis. 2005 August ; 41: 281-288.

4. Chang CM, Lu FH, Guo HR, Ko WC. Klebsiella pneumonia fascial space infections of the head and neck in Taiwan: Emphasis on diabetic patients and repetitive infections. $\mathrm{J}$ Infect. 2005; 50: 34-40.

5. Huang TT, Tseng FY, Liu TC, Hsu CT, Chen YS. Deep neck infection in diabetic patients: Comparison of clinical picture and outcomes with nondiabetic patients. Otolaryngol Head Neck Surg. 2005; 132: 943-947. 
6. Huang TT, Tseng FY, Yeh TH, Hsu CJ, Chen YS. Factors affecting the bacteriology of deep neck infection: a Retrospective study of 128 patients. ActaOtolaryngol. 2006; 126: 396-401.

7. Lee JK, Kim HD, Lim SC. Predisposing factos of complicated deep neck infection: An analysis of 158 cases. Yonsei Med J. 2007; 48(1): 55-62.

8. Park YS, Sunmi Y.Risk factors and clinical features of infections caused by plasmid-mediated AmpC $\beta$ lactamase-producing Enterobacteriaceae. Int J Antimicrob Agents. 2009; 34: 38-43.

9. Philippon A, Arlet G, Jacoby G. Plasmid-Determined AmpCType $\beta$ Lactamases. Antimicrob Agents Chemoter Jan. 2002; 46 (1): 1-11.

10. Wang JT, Chang SC, Chen YC, Luh KT. Comparison of antimicrobial susceptibility of Citrobacterfreundii isolates in two different time periods.J Microbiol. 2000; 33(4):258-62.

11. Whalen JG, Mully TW, Enlgish JC 3rd. Spontaneous Citrobacterfreundii infection in an immunocompetent patient. Archdermatol. 2007; 143(1): 124-5.

12. Kim PW, Harris AD, Roghmann MC, Morris JG Jr, Strinivasan A, Perencevich EN. Epidemiological risk factors for isolation of ceftriaxone-resistant versus -susceptible Citrobacterfreundii in hospitalized patients. Antimicrob Agents Chemother. 2003; 47(9): 2882-2887.

13. Nada T, Baba H, Kawamura K, Ohkura T, Torii K, Ohta M. A small outbreak of third generation cephem-resistant Citro- bacterfreundii infection on a surgical ward. Jpn J InfectDis. 2004; 57(4): 181-182.

14. Del Valle D. Betalactamasas tipo AmpC: Generalidades y métodos para detección fenotípica. Rev Ven SocMicriobiol. 2009; 29: 78-83.

15. Podschun R and Ullmann U. Klebsiella spp. as Nosocomial Pathogens: Epidemiology, Taxonomy, Typing Methods, and Pathogenicity Factors. ClinMicrobiol Rev. 1998; 11(4): 589-603

16. Lawlor M, Hsu J, Rick P, Miller V.Identification of Klebsiellapneumoniae virulence determinants using an intranasal infection model. MolMicrobiol. 2005; 58(4): 10541073.

17. Brisse S and Verhoef J. Phylogenetic diversity of Klebsiellapneumoniae and Klebsiellaoxytoca clinical isolates revealed by randomly amplified polymorphic DNA, gyrA and parC genes sequencing and automated ribotyping. Int $\mathrm{J}$ SystEvolMicr. 2001; 51: 915-924.

18. Wen-ChienKo, Paterson D, Sagnimeni A, Hansen D, Von Gottberg A, Mohapatra S. "Community-Acquired Klebsiellapneumoniae Bacteremia: Global Differences in Clinical Patterns". Emerg Infect Dis. 2002; 8(2): 160-166.

19. Queenan AM, Bush K. Carbapenemases: The Versatile $\beta$-lactamases. ClinMicrobiol Rev. 2007; 20(3): 440-458. 